\title{
Diabetes mellitus e hipertensión arterial en un consultorio urbano de Los Andes
}

\author{
YALDA LUCERO(1), CAROLINA GONZÁLEZ(1), GLADYS YENTZEN(2), MARÍA DE LA FUENTE(2)
}

\section{RESUMEN}

Las enfermedades cardiovasculares constituyen un problema relevante como causa de morbimortalidad. Se relacionan con varios factores de riesgo, destacando la hipertensión (HTA) $y$ diabetes (DM2). Estudios extranjeros y nacionales describen una asociación frecuente entre ambos factores. En nuestro país falta información sobre las características clínicas y demográficas de los pacientes portadores de ambas condiciones. Su objetivo es describir el perfil demográfico, clínico y factores de riesgo asociados con HTA y DM2 en un grupo de pacientes atendidos en un consultorio de Los Andes. Se revisaron las fichas de 202 pacientes con HTA y DM2, registrándose: género, edad, año de diagnóstico de HTA, DM2, obesidad y dislipidemia, peso al ingreso y actual, talla, último valor de glicemia, hemoglobina glicosilada Alc, presión arterial y perfil lipídico, tabaquismo, cumplimiento de terapia no farmacológica y fármacos indicados. El 75,7\% fueron mujeres y la edad promedio al último control fue 66,6 años, siendo el $60 \%$ mayores de 65 años. Hubo una proporción importante de pacientes con sobrepeso-obesidad (82\%) y dislipidemia $(88,1 \%)$, no así con tabaquismo (12,4\%). La proporción de descompensación fue $76,2 \%$ para DM2, $80 \%$ para HTA y $60,7 \%$ tenía ambas patologías no controladas. El 93,1\% recibia al menos 1 fármaco para tratar su HTA y DM2 y sólo en $50 \%$ se registró cumplimiento de las medidas no farmacológicas. La obesidad se relacionó en forma estadísticamente significativa con descompensación tanto de HTA como DM2, mientras que edad $<65$ años se asoció a DM2 descompensada. En este grupo, la DM2 e HTA se asociaron frecuentemente a otros factores de riesgo cardiovascular. En los pacientes obesos la tasa de compensación fue menor, lo que sugiere un comportamiento diferente de este grupo. El factor común de estas patologías sería la resistencia a insulina, que requeriría un manejo intensivo e integral desde el punto de vista del síndrome metabólico.

Palabras clave: Enfermedades cardiovasculares, Diabetes Mellitus 2, Hipertensión Arterial, Síndrome Metabólico, Atención Primaria.

\section{ABSTRACT}

DIABETES MELLITUS AND ARTERIAL HYPERTENSION IN AN URBAN LOS ANDES CLINIC

Cardiovascular diseases constitute an important problem as causes of morbimortality. They are related to various risk factors, among which arterial hypertension (AHT) and diabetes mellitus (DM2) stand out. Foreign and national studies describe a frequent association between both factors. In our country information about the clinical and demographic characteristics of

(1) Internas de Medicina $7^{\circ}$ año. Universidad de Chile. lucero yalda@yahoo.com

(2) Escuela de Salud Pública. Facultad de Medicina. Universidad de Chile. 
patients suffering from both of these conditions is lacking. The purpose of this paper is that of describing the demographic and clinical profiles, as well as risk factors associated to AHT and $D M 2$, in a group of patients receiving medical attention in a clinic of Los Andes. The medical records of 202 patients with AHT and DM2 were checked, and the following items were registered: gender, age, year of AHT diagnosis, DM2, obesity and dyslipidemia, weight at entrance into program and present weight, stature, last glycemia value, Alc glycosylated hemoglobin, arterial pressure and lipidic profile, tobaccoism, non-pharmacologic therapy observance and use of prescribed pharmaceutical products. A 75.7\% of the group were females, and the average age at the last control was 66.6 years, $60 \%$ being over 65 . An important proportion of patients were overweight-obese (82\%) and suffering from dyslipidemia (88.1\%); only $12.4 \%$ suffered from tobaccoism. The proportion of decompensation for DM 2 was $76.2 \%$, for AHT $80 \%$, and $60.7 \%$ were uncontrolled in both pathologies. A $93.1 \%$ received at least one pharmaceutical preparation for the treatment of both AHT and DM2 and only $50 \%$ were registered as fulfilling the non-pharmacologic requirements. Obesity was related in a statistically significant way to decompensation for both AHT and DM2, while age $<65$ was associated to decompensated DM2. In this group, DM2 and AHT were frequently associated to other cardiovascular risk factors. In obese patients the compensation rate was lower, a fact which suggests a different conduct in this group. The common factor of these pathologies would be the resistance to insulin, which would require an intensive and integral management from the point of view of the metabolic syndrome.

Key words: Cardiovascular diseases, Diabetes mellitus 2, Arterial hypertension, Metabolic syndrome, Primary medical care.

\section{INTRODUCCION}

A nivel mundial, las enfermedades cardiovasculares constituyen un problema de salud pública relevante, ya que son causa frecuente de morbilidad y mortalidad, consumiendo importantes recursos. En EE.UU causan cerca de 6,2 millones de hospitalizaciones anuales y son responsables de 925.000 muertes cada año, constituyendo un gasto anual de US\$ 329,2 millones ${ }^{1}$.

En Chile, 1 de cada 3 defunciones ocurre por causa cardiovascular, con una tasa de mortalidad de 149 por 100.000 habitantes ${ }^{2}$. Se estima que en nuestro país las enfermedades no transmisibles, entre las cuales las patologías cardiovasculares son las más importantes, representan el $73 \%$ de los años de vida saludables perdidos por muertes prematuras o discapacidad $^{3}$

Sin embargo, es posible realizar intervenciones de promoción y prevención debido a que estas enfermedades se relacionan con una serie de factores de riesgo, algunos de ellos modificables, como son la Diabetes
Mellitus tipo 2 (DM2), la Hipertensión Arterial (HTA), el Tabaquismo, la Dislipidemia, la Obesidad y el Sedentarismo. Existen evidencias nacionales y extranjeras de que la asociación de estos factores en pacientes con HTA aumenta la mortalidad ${ }^{4-6}$.

Según el estudio CARMEN, realizado en Valparaíso, el $47,2 \%$ de nuestra población entre 25 y 64 años tendría al menos un factor de riesgo cardiovascular, $22,3 \%$ presentaría dos factores de riesgo y $3,7 \%$ tendría tres o más factores riesgo?

Estudios chilenos han descrito una prevalencia de HTA entre $10 \%$ y $23 \%$ en la población mayor de 18 años $^{8-12}$. En lo referente a DM2, se ha reportado una prevalencia declarada de $3,2 \%$, estimándose una prevalencia real cercana al $5 \%{ }^{8}$. Estudios extranjeros han encontrado que $53-65 \%$ de los pacientes con DM 2 padecerían además de HTA ${ }^{13,14}$.

Recientemente el Ministerio de Salud (Minsal) diseñó un Programa de Salud Cardiovascular que reunió los programas de HTA y DM2. De este modo, se enfoca integralmente el problema y se optimizan recursos. Según estas pautas, 
los pacientes se clasifican en categorías de riesgo cardiovascular y de acuerdo a ello se plantean las metas y pautas de manejo2. La asociación de HTA y DM2 pone a los pacientes en la categoría de máximo riesgo cardiovascular con una probabilidad $\geq 20 \%$ de presentar un evento coronario en los siguientes diez años ${ }^{2}$.

Por ello, resulta relevante conocer las características epidemiológicas y clínicas del grupo de pacientes portadores de HTA y DM2, con el fin de planificar intervenciones racionales a nivel de atención primaria que puedan mejorar su pronóstico, optimizando los recursos.

El objetivo del presente estudio es describir el perfil demográfico, clínico y los factores de riesgo cardiovascular asociados en un grupo de pacientes portadores de HTA y DM2 atendidos en un consultorio urbano de Los Andes.

\section{MATERIAL Y MÉTODO}

Estudio descriptivo basado en la revisión de fichas médicas de pacientes portadores de HTA y DM2 atendidos en un consultorio urbano de Los Andes.

De acuerdo al censo 2002, Los Andes tiene una población de 60.198 habitantes, de cual el $92 \%$ es urbana. La población urbana beneficiaria del sistema nacional de salud es atendida por dos consultorios: uno dependiente de la municipalidad, y otro dependiente del Servicio de Salud Aconcagua. Para el presente estudio, se escogió a este último consultorio que atiende a una población estimada de 21.941 habitantes, de lo cual un $68 \%$ (14.808 hab.) es mayor de 18 años.

Los pacientes en control regular en el programa de HTA son 1.822 , con una cobertura de $68 \%$ y 570 en el programa de DM2, con $77 \%$ de cobertura. En este grupo hay 427 pacientes en control activo con diagnósticos de HTA y DM2. Se seleccionaron aleatoriamente 213 fichas ( $50 \%$ de los pacientes), de las cuales 2 no estaban disponibles, 3 se excluyeron por ser DM2 en tratamiento con insulina y 6 por abandono de controles. Se revisaron las fichas de 202 pacientes, consignando información relacionada con: género, edad en el último control, año de diagnóstico de HTA, DM2, obesidad y dislipidemia, peso al ingreso y actual, talla, último perfil lipídico, última glicemia de ayuno, hemoglobina glicosilada A $1 \mathrm{c}(\mathrm{HbAlc})$, último valor de PA, tabaquismo, cumplimiento de terapia no farmacológica y fármacos indicados.

Algunas de las fichas revisadas contaban con información sólo a partir del año 1993, lo que no permitió conocer el tiempo de exacto de evolución en los pacientes que iniciaron su cuadro antes de esa fecha. Esta limitante se resolvió clasificando a los pacientes en dos grupos, con $<10$ años $y \geq 10$ años de evolución.

Se calcularon índice de masa corporal (IMC) de ingreso, IMC actual y tiempo de evolución de HTA, DM2, dislipidemia y obesidad $(<10$ y $\geq 10$ años). De acuerdo a los criterios propuestos por Minsal y los aplicados a nivel de atención primaria (Tabla 1) se calculó la proporción de pacientes con HTA, DM2 y dislipidemia descompensada.

Tabla 1. Criterios de compensación de hipertensión arterial (HTA), diabetes mellitus 2 (DM2) y dislipidemia

\begin{tabular}{|c|c|c|}
\hline Parámetro & $\begin{array}{l}\text { Criterios Programa de } \\
\text { Salud del Adulto* }\end{array}$ & Criterios Atención Primaria \\
\hline HTA & $\mathrm{PA}<130 / 85 \mathrm{mmHg}$ & $\begin{array}{l}<65 \text { años: } \mathrm{PA}<130 / 85 \mathrm{mmHg} \\
\geq 65 \text { años: } \mathrm{PA}<140 / 90 \mathrm{mmHg}\end{array}$ \\
\hline DM2 & $\begin{array}{l}\text { glicemia }<110 \mathrm{mg} / \mathrm{dl} \text { y/o } \\
\text { HbA } 1 \mathrm{c}^{* *}<7\end{array}$ & $\begin{array}{l}<65 \text { años: glicemia }<110 \mathrm{mg} / \mathrm{dl} \text { y/o } \mathrm{HbAlc}<6,5 \\
\geq 65 \text { años: glicemia }<130 \mathrm{mg} / \mathrm{dl} \text { y/o } \mathrm{HbAlc}<7\end{array}$ \\
\hline Dislipidemia & $\begin{array}{l}\text { I.DL }<100 \mathrm{mg} / \mathrm{dl}, \mathrm{HDL} \\
>40 \mathrm{mg} / \mathrm{dl}, \mathrm{TAG}<150 \mathrm{mg} / \mathrm{dl}\end{array}$ & $\mathrm{CT}<200 \mathrm{mg} / \mathrm{dl}$ \\
\hline
\end{tabular}

*: Ministerio de Salud. Reorientación de los programas de HTA y DM. División de Rectoria y Regulación Sanitaria. Departamento de Programa de las personas. Programa de Salud del Adulto, 2002; $5-15$.

**: Hemoglobina glicosilada A lc. 
Se utilizaron los programas Microsoft Excel y Epiinfo 3.2.2 para la realización del análisis estadístico, considerando significativo un valor de $\mathrm{p}<0,05$.

\section{RESULTADOS}

\section{Características demográficas}

De los 202 pacientes, $75,7 \%$ fueron mujeres. La mediana de edad al último control fue 68 años (rango 29-91 años), sin diferencias significativas entre ambos géneros $(p=0,14)$ y el $59,9 \%$ fueron mayores de 65 años.

\section{Características de Morbilidad}

Los promedios de PA sistólica y diastólica en el último control fueron 139,9 y $82,2 \mathrm{mmHg}$ respectivamente y la glicemia fue $151,4 \pm 55,9$ $\mathrm{mg} / \mathrm{dl}$, sin diferencias significativas por género. En 2/202 pacientes no había control reciente

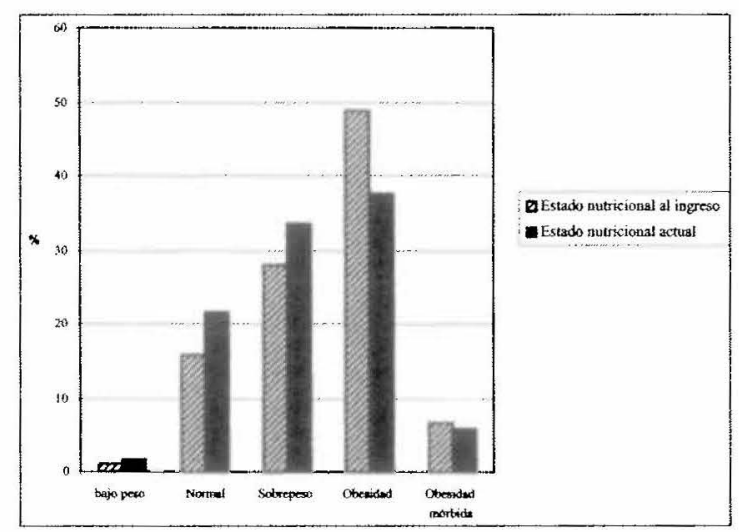

Figura 1. Distribución de los pacientes según estado nutricional (HTA) y diabetes mellitus (DM2) descompensada según a distintos factores asociados.

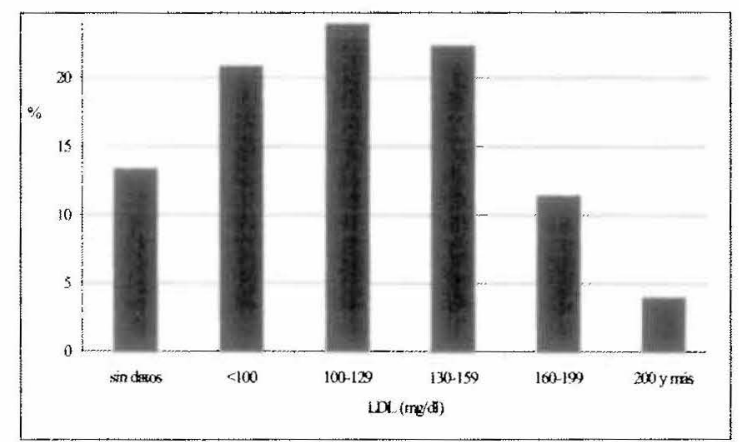

Figura 3. Distribución de los pacientes según nivel de colesterol LDL. de glicemia y se contó con valores de HbAlc sólo en $32,6 \%$ de los pacientes debido a que ésta no se solicita de rutina en este consultorio, encontrándose una mediana de $6,7 \%$.

La Figura 1 muestra la distribución según estado nutricional al ingreso y actual. El 67,2\% de los pacientes con sobrepeso, el $38,5 \%$ de los obesos y el $15 \%$ de los pacientes con obesidad mórbida al ingreso bajaron su índice ponderal (promedio $2,5 \mathrm{~kg}$ ), sin diferencias significativas en la magnitud de disminución entre los tres grupos. Según IMC en el último control el 43,2\% de los pacientes eran obesos u obesos mórbidos, sin diferencias significativas entre sexos $(37,5 \%$ hombres y $45,0 \%$ mujeres; p 0,45$)$.

El $12,4 \%$ de los pacientes tenía registrado hábito tabáquico, mientras que $52,5 \%$ no eran fumadores y en $35,1 \%$ de las fichas no se estaba consignado este dato. Hubo una mayor

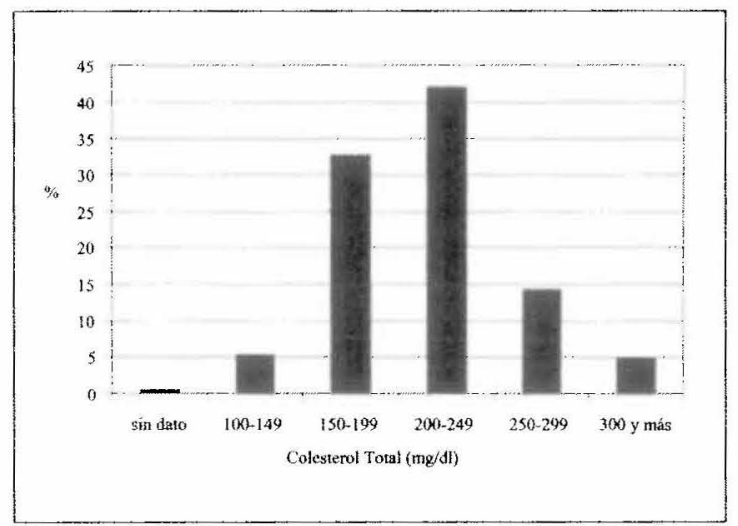

Figura 2. Distribución de los pacientes según nivel de colesterol total.

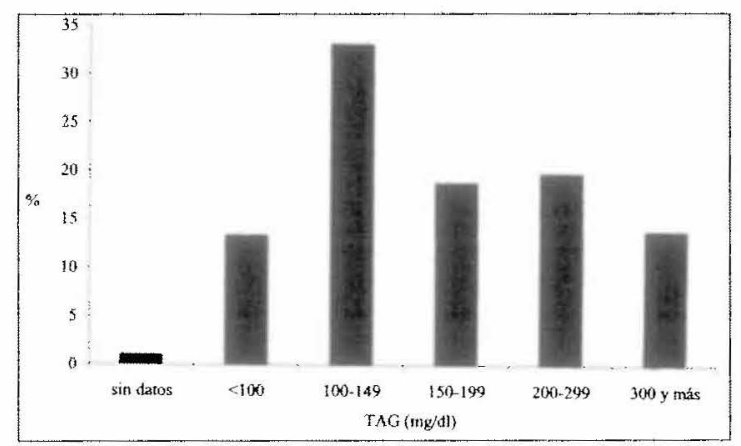

Figura 4. Distribución de pacientes segun nivel de triglicéridos (TAC). 
frecuencia de tabaquismo en los varones (p $0,015)$.

El $61,4 \%$ de los pacientes tenía diagnóstico de dislipidemia según los criterios de atención primaria $(\mathrm{CT}>200 \mathrm{mg} / \mathrm{dl})$, mientras que según las pautas del programa cardiovascular del Minsal, eran dislipidémicos el $65,8 \%$ de los pacientes por tener $\mathrm{LDL}>100 \mathrm{mg} / \mathrm{dl}, 52,5 \%$ al considerar $\mathrm{TAG}>150 \mathrm{mg} / \mathrm{dl}$ y $18,8 \%$ por tener niveles de $\mathrm{HDL}<40 \mathrm{mg} / \mathrm{dl}$, siendo en total $88,1 \%$ de los pacientes dislipidémicos.

Las Figuras 2, 3 y 4 muestran la distribución de los pacientes según nivel de CT, LDL y TAG en el último control, respectivamente.

La Tabla 2 muestra la proporción de pacientes descompensados según los criterios propuestos en el programa cardiovascular y

Tabla 2. Proporción de pacientes descompensados según tipos de criterios de evaluación

\begin{tabular}{lcc}
\hline $\begin{array}{c}\text { Criterios } \\
\text { programa } \\
\text { cardiovascular }\end{array}$ & $\begin{array}{c}\text { Criterios } \\
\text { atención } \\
\text { primaria }\end{array}$ \\
\hline Hipertensión & $80,1 \%$ & $65,7 \%$ \\
Diabetes & $76,2 \%$ & $63,7 \%$ \\
Diabetes + Hipertensión & $60,7 \%$ & $45,3 \%$ \\
\hline
\end{tabular}

los utilizados en atención primaria. Al evaluar al grupo con medición de $\mathrm{HbAlc}$, el $62,1 \%$ tenía compensada su DM2.

La Tabla 3 muestra la proporción de pacientes en que se indicó terapia no farmacológica exclusiva o junto con medicamentos para el control de la HTA y DM2. Según lo consignado en las fichas por el equipo de salud, el $50 \%$ de los pacientes no cumplía con las medidas no farmacológicas aconsejadas. Sin embargo, en el $16 \%$ de los casos, no estaba registrado este dato.

En la Tabla 4 se muestra la proporción de pacientes con HTA y DM2 descompensada de acuerdo a los criterios del Minsal, entre grupos con distintos factores asociados (género, obesidad, tiempo de evolución, edad). La obesidad se asoció en forma significativa con descompensación tanto de HTA como DM2 y la edad $<65$ años se relacionó con descompensación de DM2.

\section{DISCUSIÓN}

La asociación entre HTA y DM2 ubica a los pacientes en una categoría de máximo riesgo cardiovascular ${ }^{2}$, por lo que en este grupo es de vital importancia la pesquisa precoz y control

Tabla 3. Terapia indicada para el manejo de hipertensión arterial y diabetes en 202 pacientes

\begin{tabular}{lrrrrr}
\hline & $\begin{array}{c}\text { Sólo medidas no } \\
\text { farmacológicas }\end{array}$ & 1 fármaco & 2 fámacos & 3 fármacos & 4 fármacos \\
\hline $\begin{array}{l}\text { Hipertensión } \\
\text { Diabetes }\end{array}$ & $6,9 \%$ & $48,5 \%$ & $39,1 \%$ & $5,0 \%$ & $0,5 \%$ \\
\hline
\end{tabular}

Tabla 4. Comparación de la proporción de pacientes con hipertensión (HTA) y diabetes mellitus (DM2) descompensada según a distintos factores asociados

\begin{tabular}{lcccc}
\hline & $\begin{array}{c}\text { HTA } \\
\text { descompensada (\%) }\end{array}$ & $\mathbf{p}$ & $\begin{array}{c}\text { DM2 } \\
\text { descompensada (\%) }\end{array}$ & $\mathbf{p}$ \\
\hline Mujer & $82(54)$ & 0,2 & $115(76)$ & 0,92 \\
Hombre & $32(65)$ & $37(75)$ & 0,0052 \\
Obeso & $57(81)$ & 0,0054 & $74(86)$ & 0,24 \\
No obeso & $54(48)$ & & $76(68)$ & 0,02 \\
$<10$ años evolución & $63(51)$ & 0,085 & $112(74)$ & $40(83)$ \\
$\geq 10$ años evolución & $51(65)$ & 0,57 & $68(85)$ & $84(70)$ \\
$<65$ años edad & $47(58)$ & & & \\
\hline 65 años edad & $67(55)$ & & & \\
\hline
\end{tabular}


de otros factores de riesgo. En el presente estudio fue frecuente la asociación con obesidad y dislipidemia, no así con tabaquismo.

La mayor parte de los pacientes fueron de sexo femenino, lo que podría estar relacionado con una mayor frecuencia de consultas y/o con su incorporación a programas de seguimiento que permiten pesquisar factores de riesgo de enfermedad cardiovascular. Se encontró una proporción importante de adultos mayores, similar a lo descrito en otras series, lo que concuerda con la idea de que la prevalencia tanto de HTA como de DM2 aumenta con la edad 2,7-9,15.

Fue frecuente la obesidad en este grupo, mayor a lo descrito por Berríos y cols durante 1990 en población urbana de Santiago (13,2\% en hombres y $29,3 \%$ en mujeres) ${ }^{16}$, por Lanas y cols en empleados ${ }^{15} \mathrm{y}$ en el estudio CARMEN realizado en Valparaíso entre 1996-1997, $\left(29,5 \%\right.$ en población hipertensa) ${ }^{17}$. La proporción fue similar a la descrita por Pérez y cols en población mapuche ${ }^{18}$. Aunque hubo una mayor frecuencia en las mujeres, esta diferencia no resultó ser estadísticamente significativa como se ha reportado en otras series ${ }^{17,18}$. El grupo con menor alteración del índice ponderal, logró una mayor proporción de disminución de peso, mientras el grupo de obesos mórbidos prácticamente no presentó cambios. Esto sugiere que los pacientes con mayor índice ponderal requieren un manejo más intensivo para lograr una disminución en su peso. A nivel de atención primaria, el diagnóstico nutricional se fundamenta en la evaluación según IMC, sin embargo, estudios recientes sugieren una mejor correlación de alteraciones metabólicas y enfermedad cardiovascular con el perímetro abdominal ${ }^{13,14,19}$. Sería deseable que en atención primaria se usara este parámetro en la evaluación rutinaria de estos pacientes.

La dislipidemia fue una alteración metabólica frecuente en este grupo, similar a la descrita por Lanas y cols en empleados chilenos ${ }^{15}$ y mayor a la estimada por Berríos y cols en Santiago ${ }^{20}$ y en población general de Valparaíso en el estudio CARMEN ${ }^{7}$. Su proporción varía considerablemente según los criterios utilizados, obteniéndose un $26,6 \%$ más de pacientes dislipidémicos si se utilizan las pautas más exigentes. El parámetro del perfil lipídico más frecuentemente alterado fue el LDL, en cerca de $2 / 3$ de los pacientes, sin embargo, la mayoría no presentó una alteración severa. Dado que los fármacos del grupo de las estatinas disminuyen este tipo de colesterol y con ello el riesgo de morbimortalidad por causa cardiovascular $^{21}$, sería recomendable su uso en este grupo, que se ubica en la categoría de máximo riesgo cardiovascular. En la mitad de los casos se encontró hipertrigliceridemia y de ellos, 1/3 correspondía a una alteración severa (> 300 $\mathrm{mg} / \mathrm{dl})$. Las medidas no farmacológicas, especialmente la dieta, tendrían un impacto favorable en este grupo, por lo que sería beneficioso enfatizar el cambio de hábitos en estos pacientes.

Llama la atención la baja prevalencia de tabaquismo comparada con otros estudios que describen una frecuencia de $40-45 \%^{7,17}$, sin embargo, en una de cada tres fichas no estaba consignado el dato. Al igual que en otras series, el hábito tabáquico fue más prevalente en varones 7,15 .

Una proporción importante de pacientes tenían su HTA y DM2 descompensada, siendo significativamente mayor de acuerdo a los criterios propuestos en el programa cardiovascular del Minsal. La falta de respuesta podría deberse a la poca adherencia de los pacientes al tratamiento, especialmente a la terapia no farmacológica. Llama la atención este fenómeno, pese a la realización de reiterados controles con nutricionista y enfermera donde se hace énfasis en la importancia de estas medidas. Debido a que estos pacientes se encuentran en la categoría de máximo riesgo cardiovascular, es deseable mantener su PA y glicemia en rango normal para retrasar la aparición de complicaciones. Sería razonable por ello adoptar los criterios más estrictos de evaluación de compensación e implementar intervenciones más agresivas en el grupo de pacientes descompensados.

Por problemas de costo, sólo a $1 / 3$ de los pacientes se les solicitó $\mathrm{HbAlc}$, que es un mejor parámetro que la glicemia de ayuno para evaluar la estabilidad metabólica de la DM222. Aunque hubo una proporción importante de pacientes con HbAlc en rangos de compensación, ésta fue solicitada a pacientes 
seleccionados y, por lo tanto, este resultado no es extrapolable al resto del grupo.

Uno de cada cuatro pacientes manejaba su DM2 sólo con medidas no farmacológicas, mientras que en HTA eran pocos los que no recibían fármacos. Esto podría deberse, como se expuso anteriormente, a la baja adherencia al régimen hiposódico y/o al uso precoz de inhibidores de la enzima convertidora de angiotensina en pacientes diabéticos que presentan HTA, pues está demostrado que retarda el daño renal23.

En nuestro país, la obesidad muestra una tendencia al incremento en todos los grupos de edad, posiblemente relacionado con los cambios del patrón de alimentación de la población ${ }^{24}$. El seguimiento a largo plazo realizado en el estudio de Framingham mostró que los obesos tenían una incidencia significativamente mayor de eventos cardiovasculares importantes que los sujetos normales ${ }^{25}$. En el presente estudio, la obesidad se asoció con una mayor proporción de descompensación tanto de HTA como de DM2, lo que sugiere que el grupo de pacientes obesos, tendría un comportamiento metabólico y clínico diferente. En este contexto resulta relevante conocer el Síndrome Metabólico definido por la presencia de al menos tres de los siguientes cinco factores: Obesidad abdominal (circunferencia abdominal $>102 \mathrm{~cm}$ en hombres y $>88 \mathrm{~cm}$ en mujeres), Triglicéridos séricos (TAG) $>150 \mathrm{mg} / \mathrm{dl}$, Colesterol HDL $<40$ $\mathrm{mg} / \mathrm{dl}$ en hombres $\mathrm{y}<50 \mathrm{mg} / \mathrm{dl}$ en mujeres, Presión Arterial (PA) $\geq 130 / 85 \mathrm{~mm}$ de $\mathrm{Hg} y$ Glicemia de ayuno $\geq 110 \mathrm{mg} / \mathrm{dl}^{19}$. En su patogenia destaca la resistencia a la insulina que predispone a ateromatosis y con ello aumenta la probabilidad de sufrir un evento cardiovascular $^{13,19}$. Estudios recientes sugieren que en Estados Unidos la prevalencia del Síndrome Metabólico estaría entre $21,8 \%$ y $23 \%$ en población adulta, aumentando progresivamente con la edad, alcanzando un máximo de $42 \%$ en pacientes mayores de 60 años 26 . Hasta el momento no hay datos disponibles que permitan estimar la prevalencia de este síndrome en adultos de nuestro país.

Los datos surgidos de este estudio sugieren que el manejo de los pacientes con asociación de HTA, DM2 y obesidad requeriría un enfoque distinto, considerando en su patogenia la resistencia a insulina ${ }^{8,13}$. De ahí la importancia de incorporar los cambios de hábito, con una dieta sana y actividad física regular en este grupo de pacientes para conseguir una compensación de sus alteraciones metabólicas.

Una limitación de este estudio fue el subregistro, principalmente en lo referente al cumplimiento de la terapia y al hábito tabáquico. Sería de utilidad que en el futuro se implementara un formato estándar para registrar la información de los controles, con el objeto de facilitar esta tarea y evitar la ausencia de información relevante en el manejo de estos pacientes.

Este trabajo constituye una primera aproximación al problema de la asociación de HTA y DM2. En el futuro deberían realizarse nuevos estudios para estimar la prevalencia de esta asociación y confirmar su relación con el síndrome metabólico.

En conclusión, podemos decir que en el grupo de pacientes estudiados la obesidad y la dislipidemia se presentaron con frecuencia como factores de riesgo asociados, no así el tabaquismo. La detección de HTA y DM2 descompensada y la frecuencia de dislipidemia fue mayor al utilizar los criterios propuestos en el programa cardiovascular del Minsal que con los usados en atención primaria, por lo que sería recomendable utilizar los primeros para un manejo óptimo de este grupo de pacientes. Sólo la obesidad tuvo una asociación estadísticamente significativa con descompensación tanto de DM2 como HTA, sugiriendo un comportamiento diferente de este grupo, probablemente relacionado con el síndrome metabólico, por lo que requeririan un manejo integral del cuadro de resistencia a insulina.

\section{REFERENCIAS}

1.- AMERICAN HEART ASSOCIATION. Heart and Stroke Statistical Update. Dallas 2000. WWw americanheart.org

2. MINISTERIO DE SALUD. Reorientación de los programas de HTA y DM. División de Rectoría y Regulación Sanitaria. Departamento de Programa de las personas. Programa de Salud del Adulto, $2002 ; 5-15$.

3.- MINISTERIO DE SALUD. Estudio carga de 
enfermedad, componente cuantitativo del estudio de priorización de inversiones. Chile 1996.

4.- KANNEL, W B. Status of risk factors and their consideration in antihypertensive therapy. Am $\mathrm{J}$ Cardiol 1987; 59: 80A-90A.

5.- MULTIPLE RISK FACTOR INTERVENTION TRIAL GROUP. Exercise electrocardiogram and coronary heart disease mortality in the Multiple Risk Factor Intervention Trial. Am J Cardiol 1985; 55: 16-24.

6. - ROMÁN O, CUEVAS G, BUNOUT D. Influencia de factores de riesgo y terapia farmacológica en la mortalidad de hipertensos esenciales. Rev Méd Chile $1998 ; 126: 745-52$.

7.- JADUE L, VEGA J, ESCOBAR M, DELGADO I, GARRIDO C, LASTRA P, ESPEJO F, PERUGA A. Factores de riesgo para las enfermedades no transmisibles: Metodología y resultados globales de la encuesta de base del programa CARMEN (Conjunto de Acciones para la Reducción Multifactorial de las Enfermedades no Transmisibles). Rev Méd Chile 1999; 127: 1004-13.

8. - MINSAL. Encuesta da calidad de vida 2000. www.epi.minsal.cl

9. FASCE E, PÉREZ H, BOGGIANO G, LECAN. NELIER E. La hipertensión arterial en una comunidad urbana de Chile. Rev Chil Cardiol 1992; 11:1-12.

10.- FASCE E, PÉREZ H, BOGGIANO G, IBÁÑEZ P. NIETO C. Hipertensión arterial en comunidades rurales. Estudio en la VIII región. Rev Méd Chile $1993 ; 121: 1058-67$.

11.- VEGA J, JADUE L, ESCOBAR MC, DELGADO I, GARRIDO C, LASTRA PET AL. Prevalencia de liipertensión arterial en Valparaíso. Rev Méd Chile 1999; 127: 729-38.

12. Epidemiología de la HTA: Conclusiones del simposium del MERCOSUR. Congreso de la Soc Argentina de HTA. Bariloche 2000.

13. CONTRERAS F, RIVERA M, VASQUEZ J, DE LA PARTE M, VELASCO M. Diabetes and hypertension physiopathology and therapeutics. J Hum Hypertens, 2000; 14(Suppl 1): S26-31

14. CRESPO N, MARTINEZ A, ROSALES E, CRESPO N, GARCÍA J. Diabetes Mellitus e hipertensión. Estudio en el nivel primario de salud. Rev Cubana Med Gen Integr 2002; 18: $331-5$.

15.- LANAS F, DEL SOLAR J, MALDONADO M,
GUERRERO M, ESPINOZA F. Prevalencia de factores de riesgo de enfermedad cardiovascular en una población de empleados chilenos. Rev Méd Chile 2003; 131: 129-34.

16.- BERRÍOS X, JADUE L, ZENTENO J, ROSS M, RODRÍGUEZ H. Prevalencia de factores de riesgo de enfermedades crónicas. Estudio en población general de la región metropolitana, 1986-1987. Rev Méd Chile 1990; 118: 597-604.

17.- VEGA J, JADUE L, ESCOBAR M C, JALIL J, ESPEJO F, DELGADO I et al. Prevalencia de hipertensión arterial en Valparaíso. Resultados de la encuesta de base del programa CARMEN (Conjunto de acciones para la reducción multifactorial de las enfermedades no transmisibles). Rev Méd Chile 1999; 127: 729-38.

18.- PÉREZ F, CARRASCO E, SANTOS J, CALVILLÁN M, ALBALA C. Prevalencia de obesidad, hipertensión arterial y dislipidemia en grupos aborígenes rurales de Chile. Rev Méd Chile 1999; 127: 1169-75.

19.- ROBERTS W. The Metabolic Syndrome. Am J Cardiol 2004; $93: 274$

20.- BERRÍOS $X$, JADUE $L$, PIEROTIC M. Perfil lipídico en la población adulta de la región metropolitana. Rev Méd Chile 1992; 120: $331-3$.

21.- ARONOW W S. Hypercholesterolemia: The evidence supports use of statins. Geriatrics 2003; 58(August): 18-32.

22.- MIEDEMA K, CASPARIE T. Glycosylated haemoglobins: biochemical evaluation and clinical utility. Ann Clin Biochem 1984; 21 (Pt 1): 2-15.

23.- BALL S. Benefits of blood pressure reduction in diabetic patients. J Hypertens. 2003; 21 (Suppl 6): \$31-6.

24.- ALBALA C, VIOF, KAIN J. Obesidad: un desafío pendiente en Chile. Rev Méd Chile 1998; 126: $1001-9$.

25.-HUBERT H, FEINLEB M, MCNAMARA P et al. Obesity as an independant risk for cardiovascular disease: a 26 years follow up of participants in the Framingham Heart Study. Circulation 1983; 67: $968-77$.

26. FORD E, GILES W, DIETZ W. Prevalence of the metabolic syndrome among US adults: findings from the third National Health and Nutrition Examination Survey. JAMA, 2002; $287(3): 356-9$.

\section{Anexo: Definiciones Operacionales}

HTA: PA sistólica $\geq 140 \mathrm{mmHg}$ y/o PA diastólica $\geq 90 \mathrm{mmHg}$ en al menos 2 de 3 tomas seriadas en días diferentes.

DM2: glicemia $\geq 200 \mathrm{mg} / \mathrm{d} 1$ y síntomas de descompensación o glicemia $\geq 126 \mathrm{mg} / \mathrm{d}$ l aislada o glicemia $\geq 200 \mathrm{mg} /$ dl a las 2 horas post carga de $75 \mathrm{~g}$ de glucosa (PTGO).

Estado nutricional:

Sobrepeso: en $<65$ años IMC 25-29. En $\geq 65$ años IMC 27-31.

Obesidad: en $<65$ años $I M C \geq 30$. En $\geq 65$ años $I M C \geq 32$.

Dislipidemia: colesterol total $(\mathrm{CT}) \geq 200 \mathrm{mg} / \mathrm{dl} / \mathrm{o} \mathrm{LDL} \geq 100 \mathrm{mg} / \mathrm{dl} \mathrm{y} / \mathrm{HDL}<40 \mathrm{mg} / \mathrm{dl} \mathrm{y} / \mathrm{o}$ triglicéridos (TAG) $\geq 150 \mathrm{ing} / \mathrm{dl}$. 